\title{
MACHINE-LEARNING ESTIMATION OF BODY Posture and Physical ACTIVITY BY Wearable ACCELERATION AND HEARTBEAT SENSORS
}

\author{
Yutaka Yoshida ${ }^{2}$, Emi Yuda $^{3,1}$, Kento Yamamoto ${ }^{4}$, Yutaka Miura ${ }^{5}$, and \\ Junichiro Hayano ${ }^{1}$ \\ ${ }^{1}$ Department of Medical Education, Nagoya City University Graduate School of Medical \\ Science, Nagoya, Japan \\ ${ }^{2}$ Nagoya City University Graduate School of Design and Architecture, Nagoya, Japan \\ ${ }^{3}$ Department of Electrical Engineering, Tohoku University Graduate School of \\ Engineering, Sendai, Japan \\ ${ }^{4}$ University of Tsukuba Graduate School of Comprehensive Human Sciences, \\ Tsukuba, Japan \\ ${ }^{5}$ Department of Nutrition, Shigakkan University, Obu, Japan
}

\begin{abstract}
We aimed to develop the method for estimating body posture and physical activity by acceleration signals from a Holter electrocardiographic (ECG) recorder with built-in accelerometer. In healthy young subjects, triaxial-acceleration and ECG signal were recorded with the Holter ECG recorder attached on their chest wall. During the recording, they randomly took eight postures, including supine, prone, left and right recumbent, standing, sitting in a reclining chair, sitting in chairs with and without backrest, and performed slow walking and fast walking. Machine learning (Random Forest) was performed on acceleration and ECG variables. The best discrimination model was obtained when the maximum values and standard deviations of accelerations in three axes and mean $R-R$ interval were used as feature values. The overall discrimination accuracy was $79.2 \%$ (62.6-90.9\%). Supine, prone, left recumbent, and slow and fast walk were discriminated with $>80 \%$ accuracy, although sitting and standing positions were not discriminated by this method.
\end{abstract}

\section{KEYWORDS}

Accelerometer, Holter ECG, Posture, Activity, Machine learning, Random Forest, R-R interval

\section{INTRODUCTION}

The importance of physical activity for health maintenance and promotion is gathering increasing attention. Sedentary behaviour is known to be associated with the incidence of malignant arrhythmias, and there is accumulating evidence to support that the maintenance and increase of physical activity decrease the risk of sudden cardiac death [1]. Increased physical activity by exercise can be expected to be effective in lowering the functional level of daily life and in preventing life-style related diseases including diabetes, dyslipidaemias, metabolic syndrome, heart diseases, and malignancies [2]. The World Health Organization's Global Recommendation for Physical Activity [1] states an increase in total step counts in daily life as a goal in the field of daily physical activity and exercise. It says that an increase of 1500 steps a day accounts for $2 \%$ reduction in the incidence and mortality by non-communicable diseases and $1.5-\mathrm{mm} \mathrm{Hg}$ reduction in blood pressure. 
Even though these required physical activity criteria have been proposed based on scientific evidence, the information used to assess physical activity is often depends on that obtained by conventional methods such as a pedometer, in which information such as the type and frequency of postural changes and the speed of action and movement is ignored. As a result, the adjustment for the effects of age, gender, weight, and height on the assessment of physical activity, if implemented, become less rigorous.

To overcome this problem, several studies proposed acceleration and other sensors [3-10] and algorithms including machine learning for the precise estimation of body posture and the type and level of physical activity [6,7,10]. The reported performance, however, differed with the type, number, and placement of sensors, signal sampling frequency, analysis window size, machine learning technique, and measurement setting, i.e., laboratory or free-living conditions [11]. In this study, we investigated the posture and activity estimation performance of a built-in accelerometer of a chest-mounted Holter electrocardiographic (ECG) recorder widely used for clinical purposes in combination with the Random Forest machine learning technique.

\section{EXPERIMENT METHODS}

\subsection{Subjects}

We studied 11 healthy subjects ( 2 men, 9 women, $22 \pm 1$ yr) who gave a written informed consent to participate this study. The protocol of the present study was approved by the Institutional Review Board of Nagoya City University Graduate School of Medical Sciences and Nagoya City University Hospital (approval number, 60-18-0211).

\subsection{Measurement device}

Holter ECG recorders with a built-in 3-axis acceleration sensor (Cardy 303 pico+, Suzuken Co., Ltd; size, W28 $\times$ D $42 \times \mathrm{H} 9 \mathrm{~mm}$, weight, $13 \mathrm{~g}$ ) were used for measuring acceleration and ECG signals. The sampling frequency of the ECG was $128 \mathrm{~Hz}$. The accelerometer recorded acceleration from left to right, cranio-caudal, and postero-anterior directions as the $\mathrm{X}, \mathrm{Y}$, and $\mathrm{Z}$ values, respectively, at a frequency of $31.25 \mathrm{~Hz}$.

\subsection{Experimental protocol}

Experiments were conducted in an air-conditioned laboratory at $24 \pm 2{ }^{\circ} \mathrm{C}$. The Holter ECG recorder and electrodes were attached on subjects' chest wall at 09:30. Subjects were instructed to take supine, prone, right and left recumbent, and standing positions, to sit in three different chairs (a recliner and chairs with and without a backrest), and to walk slowly and walk fast, in a randomized order. Subjects maintained each posture and activity for $110 \mathrm{~s}$, and then moved to the next posture or activity within $10 \mathrm{~s}$.

\subsection{Data analysis}

\subsubsection{Analysis of 3-axis acceleration time series and RRI time series}

The acceleration signals in $\mathrm{X}, \mathrm{Y}$, and $\mathrm{Z}$ axes at $31.25 \mathrm{~Hz}$ were under-sampled at $2 \mathrm{~Hz}$ per axis, and average, median, maximum, minimum, and standard deviation (SD) were calculated for every $10 \mathrm{~s}$ for each axis. Because the acceleration data were unstable during the beginning of each posture and activity, the first $20 \mathrm{~s}$ of each posture was removed, resulting in nine 10-s segments per posture/activity. 
From the ECG signal, all $\mathrm{R}$ waves (sharp deflections corresponding to the ventricular electrical excitation) were detected and an R-R interval time series were obtained. R-R intervals consisting of consecutive sinus rhythms were extracted, resampled at $2 \mathrm{~Hz}$ using a step function, and averaged over every $10 \mathrm{~s}$.

\subsubsection{Feature Selection}

As the feature datasets of acceleration variables for machine learning, we examined (1) the averages and SDs of 3 axes, (2) the medians and SDs of 3 axes, (3) the maximums and SDs of 3 axes, (4) the minimums and SDs, and (5) all indices of 3 axes. In addition, the average of the R-R interval was added to each of the acceleration datasets, yielding 10 feature datasets. The number of data for one posture was nine (90 seconds) per variable. The data for sitting positions in different chairs were put together and nine time points were randomly selected from the pooled dataset.

\subsubsection{Machine learning of classification and verification}

We used Random Forest for the machine learning classifier. To reduce the bias of the learning data, learning and verification were performed using $\mathrm{k}$-fold cross validation method $(\mathrm{k}=11)$. There was no overlap between the training and the test data. These processes were performed with Python (3.6) obtained from the open data science platform Anaconda (ANACONDA, https://www.anaconda.com/).

Verification of discrimination was performed by calculating the true positive (TP) and false negative (FN) for each posture from each subject, and then adding the TP and the FN from all subjects. The recall rate defined as equation (i) was calculated for each posture. For example, in the discrimination of the supine position, the TP was the number of successful discriminations of supine and FN was the number of unsuccessful discriminations of supine.
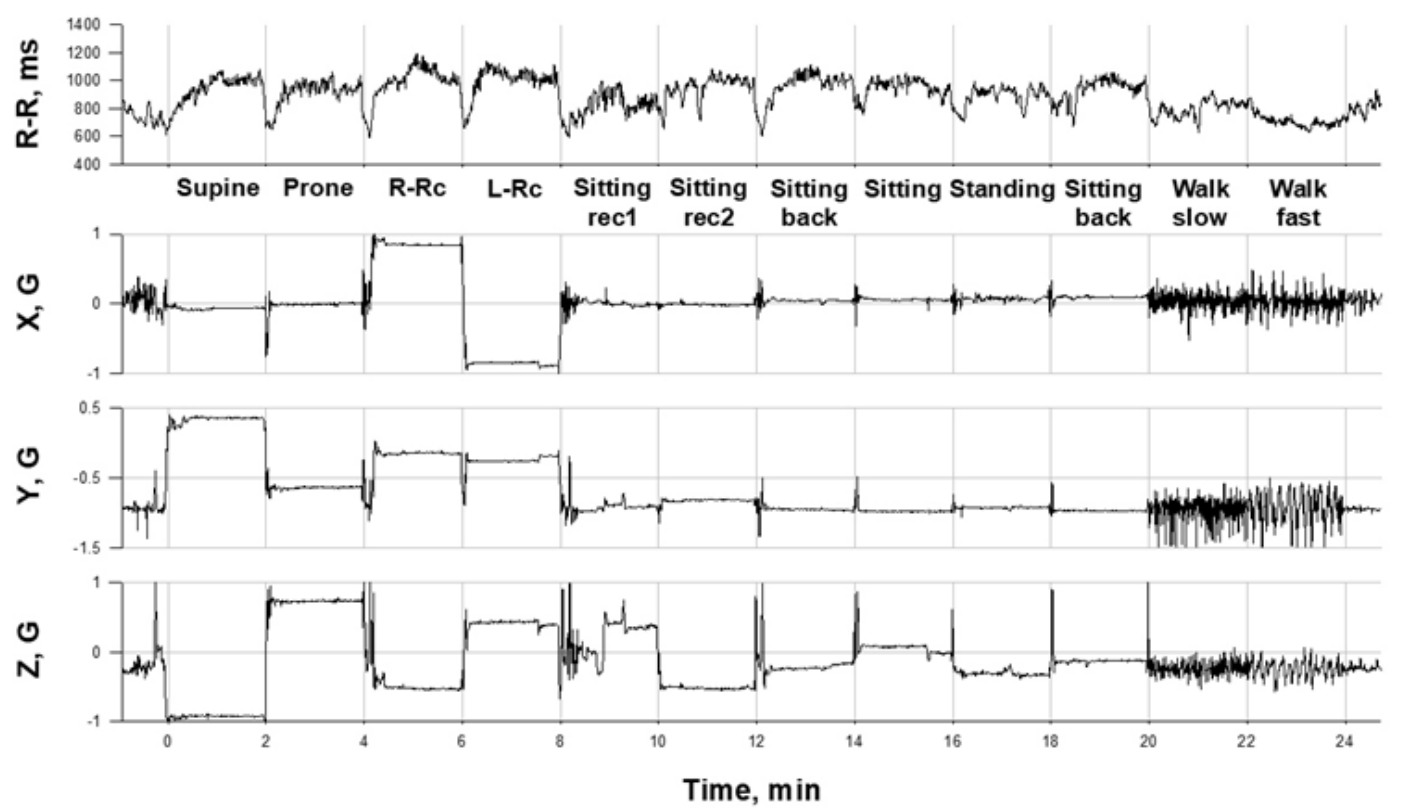

Fig. 1. Time series of R-R interval and 3-axis acceleration during experiment.

$\mathrm{R}-\mathrm{Rc}=$ Right recumbent, $\mathrm{L}-\mathrm{Rc}=$ Left recumbent, Sitting rec1 $=$ sitting in a recliner with knee extending, Sitting rec2 $=$ sitting in a recliner with knee bending, Sitting back $=$ sitting in a chair with a backrest. 
Signal \& Image Processing: An International Journal (SIPIJ) Vol.10, No.3, June 2019

Table 1. Recall ratio for posture with feature dataset of acceleration

\begin{tabular}{llllll}
\hline \multirow{2}{*}{ Posture/activity } & \multicolumn{2}{l}{ Acceleration } & & \\
\cline { 2 - 6 } & Average SD & Median SD & Max SD & Min SD & All indices \\
\hline Spine & 0.869 & 0.859 & 0.859 & 0.859 & 0.869 \\
Prone & 0.798 & 0.788 & 0.717 & 0.778 & 0.657 \\
R-recumbent & 0.727 & 0.707 & 0.747 & 0.747 & 0.727 \\
L-recumbent & 0.909 & 0.909 & 0.909 & 0.919 & 0.909 \\
Sitting & 0.636 & 0.646 & 0.616 & 0.586 & 0.747 \\
Standing & 0.626 & 0.576 & 0.616 & 0.606 & 0.535 \\
Slow walk & 0.677 & 0.677 & 0.768 & 0.727 & 0.687 \\
Fast walk & 0.697 & 0.747 & 0.758 & 0.677 & 0.758 \\
\hline Accuracy & 0.742 & 0.739 & 0.749 & 0.737 & 0.736 \\
SD & 0.106 & 0.111 & 0.103 & 0.116 & 0.118 \\
\hline
\end{tabular}

Accuracy was calculated as the mean value of recalls for all postures.

$\mathrm{SD}=$ standard deviation

Table 2. Recall ratio for posture with feature dataset of acceleration and R-R interval

\begin{tabular}{llllll}
\hline \multirow{2}{*}{ Posture/activity } & Acceleration & & & \\
\cline { 2 - 6 } & Average SD & Median SD & Max SD & Min SD & All indices \\
\hline Spine & 0.869 & 0.838 & 0.899 & 0.879 & 0.859 \\
Prone & 0.818 & 0.859 & 0.859 & 0.859 & 0.636 \\
R-recumbent & 0.747 & 0.727 & 0.737 & 0.737 & 0.747 \\
L-recumbent & 0.909 & 0.859 & 0.909 & 0.919 & 0.909 \\
Sitting & 0.758 & 0.667 & 0.717 & 0.667 & 0.768 \\
Standing & 0.525 & 0.586 & 0.626 & 0.535 & 0.434 \\
Slow walk & 0.657 & 0.606 & 0.818 & 0.687 & 0.828 \\
Fast walk & 0.717 & 0.737 & 0.768 & 0.758 & 0.737 \\
\hline Accuracy & 0.750 & 0.735 & 0.792 & 0.755 & 0.740 \\
SD & 0.122 & 0.110 & 0.098 & 0.128 & 0.149 \\
\hline
\end{tabular}

Accuracy was calculated as the mean value of recalls for all postures.

$$
\text { Recall }=\mathrm{TP} /(\mathrm{TP}+\mathrm{FN}) \quad \cdots
$$

The number of the decision tree and the maximum depth of the tree of the Random Forest were 3 and 20 , respectively, and the maximum value of the recall was calculated by the grid search function in this range. 


\section{RESULTS}

Fig. 1 shows the examples of R-R interval and 3-axis acceleration time series during the experiment. The acceleration signals changed according to the changes in the relative direction of gravitation vector with postures. In addition, the acceleration signals, particularly in Y-axis reflecting cranio-caudal direction, showed fluctuations during walking and its amplitude was greater for fast walk than slow walk.

Table 3. Confusion matrix of feature dataset with the best classification performance

\begin{tabular}{|c|c|c|c|c|c|c|c|c|c|c|c|}
\hline \multirow{2}{*}{\multicolumn{2}{|c|}{ Posture/activity }} & \multicolumn{8}{|c|}{ Estimated posture/activity } & \multirow{2}{*}{ Segment } & \multirow{2}{*}{ Recal } \\
\hline & & $\mathrm{Su}$ & $\operatorname{Pr}$ & $\mathrm{Rr}$ & $\mathrm{Lr}$ & $\mathrm{Si}$ & $\mathrm{St}$ & $\mathrm{Sw}$ & Fw & & \\
\hline \multirow{8}{*}{ Actual } & $\mathrm{Su}$ & 89 & 0 & 7 & 0 & 0 & 0 & 1 & 2 & 99 & 0.899 \\
\hline & $\operatorname{Pr}$ & 1 & 85 & 4 & 7 & 1 & 0 & 1 & 0 & 99 & 0.859 \\
\hline & $\mathrm{Rr}$ & 7 & 15 & 73 & 1 & 3 & 0 & 0 & 0 & 99 & 0.737 \\
\hline & $\mathrm{Lr}$ & 0 & 2 & 7 & 90 & 0 & 0 & 0 & 0 & 99 & 0.909 \\
\hline & $\mathrm{Si}$ & 2 & 0 & 0 & 0 & 71 & 26 & 0 & 0 & 99 & 0.717 \\
\hline & St & 0 & 0 & 0 & 0 & 34 & 62 & 3 & 0 & 99 & 0.626 \\
\hline & Sw & 0 & 0 & 0 & 0 & 0 & 1 & 81 & 17 & 99 & 0.818 \\
\hline & FW & 0 & 0 & 0 & 0 & 0 & 0 & 23 & 76 & 99 & 0.768 \\
\hline
\end{tabular}

$\mathrm{Su}=$ supine, $\mathrm{Pr}=$ prone, $\mathrm{Rr}=$ right recumbent, $\mathrm{Lr}=$ left recumbent, $\mathrm{Si}=$ sitting, $\mathrm{St}=$ standing, $\mathrm{Sw}$ = slow walk, Fw fast walk.

Table 4. Gross confusion matrix for coarse-grained posture/activity

\begin{tabular}{llllllll}
\hline \multirow{2}{*}{ Posture/activity } & \multicolumn{2}{l}{ Estimated } & & & \multirow{2}{*}{ Segment } & \multirow{2}{*}{ Recall } \\
\cline { 2 - 6 } & Lying & Sitting & Standing & Walking & & \\
\hline \multirow{4}{*}{ Actual } & Lying & 388 & 4 & 0 & 4 & 396 & 0.980 \\
& Sitting & 2 & 71 & 26 & 0 & 99 & 0.717 \\
& Standing & 0 & 34 & 62 & 3 & 99 & 0.626 \\
& Walking & 0 & 0 & 1 & 197 & 198 & 0.999 \\
\hline Segment & 390 & 109 & 89 & 204 & 792 & \\
Specificity & 0.995 & 0.945 & 0.961 & 0.986 & Accuracy & \\
Positive predictive value & 0.995 & 0.651 & 0.697 & 0.966 & 0.907 & \\
\hline
\end{tabular}

Data are classification results by feature dataset with the maximum and SD of acceleration and mean R-R interval.

Tables 1 and 2 shows the recall rate for the posture/activity by each feature dataset. The feature dataset with the highest mean recall rate was a combination of the maximum and SD of acceleration and mean R-R interval (mean recall rate $\pm \mathrm{SD}, 0.792 \pm 0.098$ ). Supine, prone, left recumbent, and slow walk were discriminated by recall rates $>0.80$, while the recall rate for standing was 0.626 .

Tables 3 and 4 shows the confusion matrix for the feature dataset with the best performance (maximum and SD of acceleration and mean R-R interval). When the postures and activities were coarse-grained into lying, sitting, standing, and walking, the overall accuracy of classification was 0.907 . The recall ratio, specificity, and positive predictive value for classifications of lying and walking were $>0.95$. 

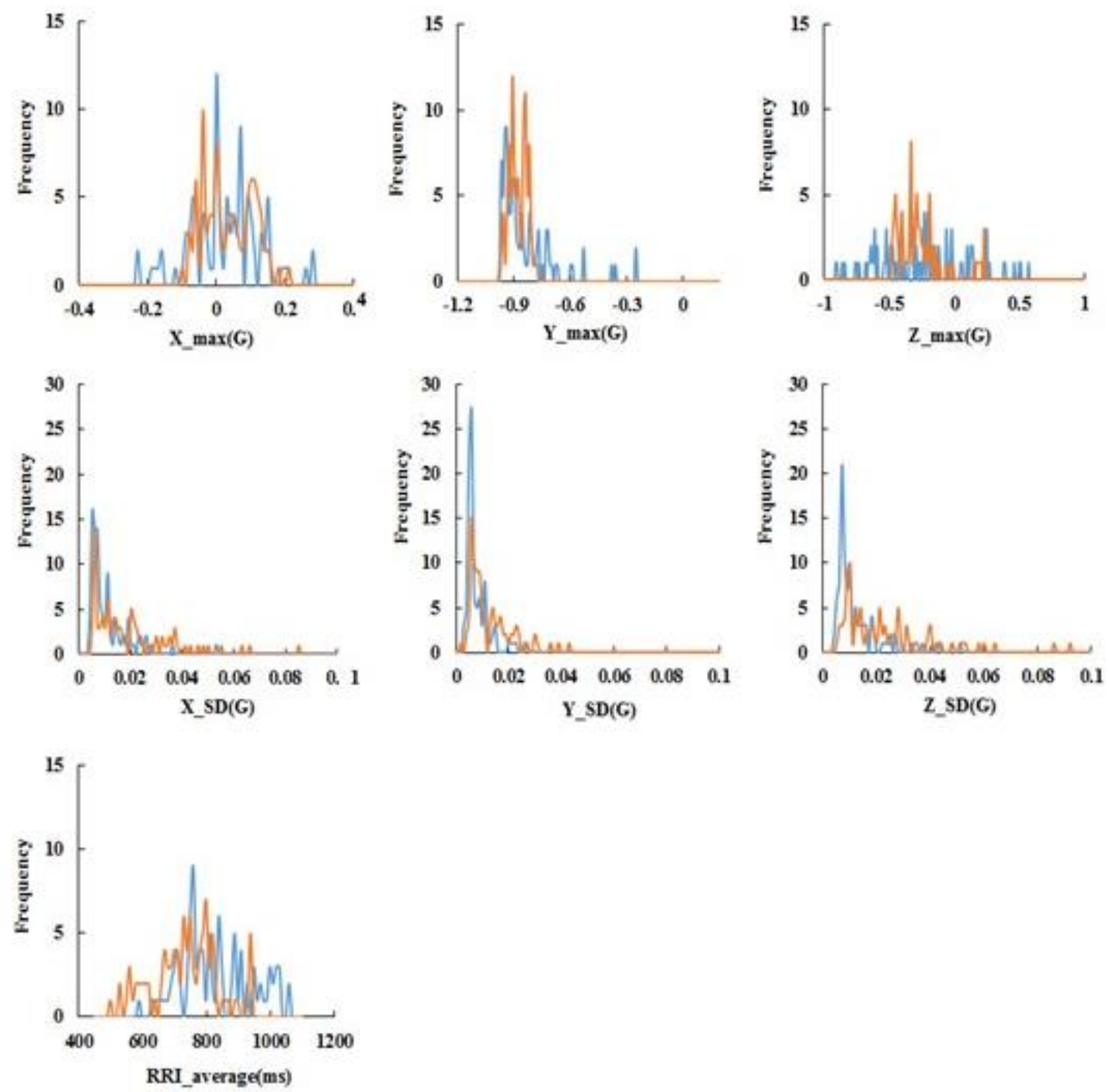

Fig. 2. Histogram of the maximum and SD of acceleration and R-R interval for sitting and standing positions.

Blue: sitting, Orange: standing.

However, the discrimination between sitting and standing were less accurate. Although out of 99 segments in sitting 71 segments were correctly discriminated as sitting, 26 segments were classified as standing, and 2 as prone position, indicating that $26 \%$ of sitting was estimated as standing. Similarly, out of 99 segments in standing 62 segments were correctly discriminated as standing, but 34 segments were classified as sitting and 3 as slow walking, indicating that standing was estimated as sitting at $34 \%$ probability. Fig. 3 is a histogram of the maximum and $\mathrm{SD}$ of acceleration and RRI in the sitting and standing postures. Both show the similar patterns of distribution. 


\section{DISCUSSIONS}

In the present study, we examined the usefulness of built-in triaxial accelerometer of a chestmounted Holter ECG recorder in combination with machine learning for estimating body posture and activity. All possible combinations of variables derived from triaxial acceleration signals every $10 \mathrm{~s}$ were used as feature dataset and discriminant models were generated by the Random Forest algorithm. The best classification performance was obtained by the feature dataset consisting of the maximum and SD of acceleration. For all feature datasets of acceleration variables, addition of mean $\mathrm{R}-\mathrm{R}$ interval improved the classification performance. By the model using the maximum and SD of acceleration and mean R-R interval classified lying, sitting, standing, and walking with an overall accuracy of 0.907 . The model showed, however, lesser discrimination performances for sitting and standing than those for lying and walking. While the recall ratios for lying and walking were 0.980 and 0.999 , the ratios for sitting and standing were 0.717 and 0.626 , respectively. Our observations show both the strengths and weaknesses of posture/activity estimation by acceleration signals and seem to suggest hints for improving the estimation.

There are many studies of the sensors and signal processing algorithms to discriminate body postures [3-10]. They reported different performance depending on factors related to sensors, signal processing, data analysis including machine learning techniques, and measurement settings. As expected, using multiple sensors gives better performance than a single sensor. Yeoh et al. [3] used 3 accelerometers at the waist and both thighs and achieved an overall accuracy of $100 \%$ for classification of lying, sitting, standing, and walking and an overall mean-square error of $1.76 \mathrm{~km} / \mathrm{h}$ for walking and running speeds. As to the type of sensor, Godfrey et al. [4] used chest mounted tri-axial accelerometer sensitive to both static and dynamic acceleration and reported sensitivity and specificity of $>0.83$ to detect activity types and postural transitions between sitting and standing using a sophisticated algorithm of Velocity Estimate and Scalar Product Activity. As to sensor placement, Fulk et al. [5] used acceleration and pressure sensors built into the shoe and successfully distinguished between sitting and standing with an accuracy of $82-100 \%$ in patients after stroke. Also, Doulah et al. [7] reported the detection of sit-to-stand posture transitions by orthosis-mounted sensors and Fanchamps et al. [9] used a acceleration sensor attached to the thigh in post-stroke patients. To discriminate activity types and postures, machine learning-based approaches are employed in most of recent studies, such as artificial neural network [7], support vector machines [5], Random Forest [10], Naïve Bayes, and Knearest neighbours [11]. Finally, as to measurement setting, the reported performance of activity and posture discriminations obtained in free-living settings were generally lower than those obtained in laboratory settings $[9,11]$. In the present study, we used a simple tri-axial static acceleration sensor built in a chest-mounted Holter ECG recorder, measured data in a laboratory setting, and generated the discriminant model for posture and activity by Random Forest technique. Thus, it is difficult to compare the present results with those of earlier studies, but the discriminant accuracies were comparable to them except that between sitting and standing.

The low discrimination accuracy between sitting and standing may be attributable to the use of chest-mounted accelerometer that was less sensitive to dynamic acceleration and insensitive to the changes in angular velocity. Although the gravity vector is almost vertical in the sitting position, it may shift backward to some extent depending on the type of chair (more precisely, on the angle at which the body leans on the backrest). In contrast, the gravity vector in the standing posture is vertical theoretically; however, since the Holter ECG recorder is worn on the inclination of the chest, some backward inclination occurs. During standing acceleration may fluctuate slightly due to body sway, while it may more stable during sitting. In this experiment, however, the fluctuation of acceleration signal was observed also during sitting (Fig. 3). In 
addition, the backward movement of the vertical acceleration vector may also occur due to walking and other forward movements, further complicating the determination. To overcome this problem, not only static features of acceleration such as mean, SD but also dynamic features of acceleration such as the pattern of changes seem necessary.

To our knowledge, there was no study to report posture discrimination using both acceleration and R-R interval. Although the discrimination accuracy differed depending on the selection of the feature dataset, the discrimination accuracy was consistently improved by combining 3-axis acceleration and R-R interval. Since R-R interval is expected to shorten with standing [12], mean $\mathrm{R}-\mathrm{R}$ interval may be useful feature to discriminate sitting and standing. This suggests possibility of using the indices of heart rate variability as the additional feature to discriminate posture/activity. Particularly, low frequency to high frequency power ratio may be useful for the feature to detect standing $[13,14]$.

\section{Conclusions}

We examined the posture and activity discrimination performance of signals from a built-in triaxial acceleration sensor in a chest-mounted Holter ECG recorder using Random Forest machine learning technique. We observed acceptable discriminate accuracy of activities and postures except for that between sitting and standing. Additionally, we found that the inclusion of R-R interval data as a feature improves the discriminatory accuracy. Because Holter ECG recorders with built-in accelerometer are widely used clinically, our findings seem to provide useful insight into the benefits and limitations of the clinical use of the accelerometer signal.

\section{REFERENCES}

[1] World Health Organization, Global recommendations on Physical Activity for Health. Geneva: World Health Organization; 2010.

[2] Sofi, F., Valecchi, D., Bacci, D., Abbate, R., Gensini, G. F., Casini, A., Macchi, C. (2011) "Physical activity and risk of cognitive decline: a meta-analysis of prospective studies", J. Intern. Med., Vol. 269, No. 1, 107-117.

[3] Yeoh, W. S., Pek, I., Yong, Y. H., Chen, X., Waluyo, A. B. (2008) "Ambulatory monitoring of human posture and walking speed using wearable accelerometer sensors", Conf Proc IEEE Eng Med Biol Soc, Vol. 2008, No., 5184-5187.

[4] Godfrey, A., Bourke, A. K., Olaighin, G. M., van de Ven, P., Nelson, J. (2011) "Activity classification using a single chest mounted tri-axial accelerometer", Med. Eng. Phys., Vol. 33, No. 9, 1127-1135.

[5] Fulk, G. D., Sazonov, E. (2011) "Using sensors to measure activity in people with stroke", Top Stroke Rehabil, Vol. 18, No. 6, 746-757.

[6] Palmerini, L., Rocchi, L., Mellone, S., Valzania, F., Chiari, L. (2011) "Feature selection for accelerometer-based posture analysis in Parkinson's disease", IEEE Trans Inf Technol Biomed, Vol. 15, No. 3, 481-490.

[7] Doulah, A., Shen, X., Sazonov, E. (2017) "Early Detection of the Initiation of Sit-to-Stand Posture Transitions Using Orthosis-Mounted Sensors", Sensors, Vol. 17, No. 12.

[8] Vaha-Ypya, H., Husu, P., Suni, J., Vasankari, T., Sievanen, H. (2018) "Reliable recognition of lying, sitting, and standing with a hip-worn accelerometer", Scand. J. Med. Sci. Sports, Vol. 28, No. 3, 1092-1102. 
[9] Fanchamps, M. H. J., Horemans, H. L. D., Ribbers, G. M., Stam, H. J., Bussmann, J. B. J. (2018) "The Accuracy of the Detection of Body Postures and Movements Using a Physical Activity Monitor in People after a Stroke", Sensors, Vol. 18, No. 7.

[10] Kerr, J., Carlson, J., Godbole, S., Cadmus-Bertram, L., Bellettiere, J., Hartman, S. (2018) "Improving Hip-Worn Accelerometer Estimates of Sitting Using Machine Learning Methods", Med. Sci. Sports Exerc., Vol. 50, No. 7, 1518-1524.

[11] Farrahi, V., Niemela, M., Kangas, M., Korpelainen, R., Jamsa, T. (2019) "Calibration and validation of accelerometer-based activity monitors: A systematic review of machine-learning approaches", Gait Posture, Vol. 68, No., 285-299.

[12] Olufsen, M. S., Tran, H. T., Ottesen, J. T., Research Experiences for Undergraduates, P., Lipsitz, L. A., Novak, V. (2006) "Modeling baroreflex regulation of heart rate during orthostatic stress", Am J Physiol Regul Integr Comp Physiol, Vol. 291, No. 5, R1355-1368.

[13] Hayano, J., Mukai, S., Fukuta, H., Sakata, S., Ohte, N., Kimura, G. (2001) "Postural response of lowfrequency component of heart rate variability is an increased risk for mortality in patients with coronary artery disease", Chest, Vol. 120, No., 1942-1952.

[14] Yoshida, Y., Furukawa, Y., Ogasawara, H., Yuda, E., Hayano, J. Longer lying position causes lower $\mathrm{LF} / \mathrm{HF}$ of heart rate variability during ambulatory monitoring. Paper presented at: 2016 IEEE 5th Global Conference on Consumer Electronics (GCCE); 11-14 Oct 2016, 2016; Kyoto, Japan.

\section{AUTHORS}

Yutaka Yoshida studied the business administration and computer science at Aichi Institute of Technology and received Ph.D. degree in 2008. He was a project researcher at Knowledge Hub of Aichi from 2011 to 2015 and was a researcher at Nagoya City University Graduate School of Medical Sciences from 2016 to 2017. Since 2018, he has been a researcher at Nagoya City University Graduate School of Design and Architecture. His specialized field is biological information engineering, signal processing and ergonomics. He received the paper award at the Japan Society of Neurovegetative Research in 2005 and 2007.

Emi Yuda was born in Tokyo, Japan in 1980. She studied informatics at M.V. Lomonosov Moscow State University until 2003 and then received M.S. degree from Tsukuba University, Japan. She received Ph.D. from Nihon University in 2019. From 2013 to 2014 she was a research assistant at Santa Monica College in California, USA. From 2015 to 2019, she was a NEDO project researcher in Nagoya City University Graduate School of Medical Sciences. Since 2019, she has been an assistant professor in Tohoku University Graduate School of Engineering. Her currently research is Medical Informatics and Data Science. She has many achievements in field of Informatics and Big Data.

Junichiro Hayano graduated Nagoya City University Medical School, Nagoya, Japan and received M.D. degree in 1980. From 1981 to 1983, he received residency trainings of psychosomatic medicine in Kyushu University School of Medicine, Fukuoka, Japan. He obtained Ph.D. degree (Dr. of Medical Science) in 1988 from Nagoya City University Graduate School of Medical Sciences. From 1990 to 1991, he was working as a visiting associate at the Behavioral Medicine Research Center, Duke University Medical Center, Durham, NC, USA. In 1984, he got a faculty position at Nagoya City University Medical

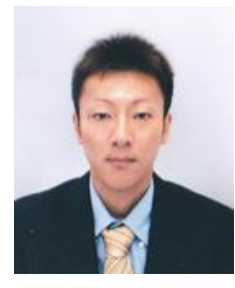

School and has been a Professor of Medicine at Nagoya City University Graduate School of Medical Sciences since 2003. His current interests are applications of dynamic electrocardiography and bio-signal processing to health sciences. 